\section{Next-generation sequencing and norovirus}
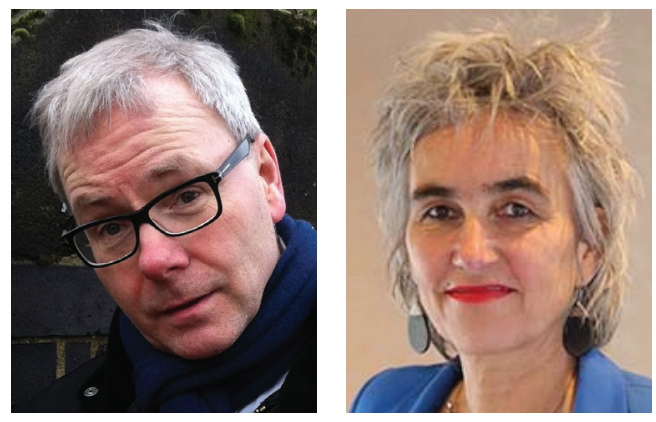

\author{
"Given the ease of generating full \\ genomes from small amounts of \\ clinical diagnostic samples and falling \\ prices of next-generation sequencing \\ methods, generating sequence data \\ from well-designed large sample \\ studies is now feasible."
}

Matthew Cotten ${ }^{1} \&$ Marion Koopmans ${ }^{*, 1}$

First draft submitted: 23 September 2016; Accepted for publication: 23 September 2016; Published online: 11 November 2016

Noroviruses (NoVs) are highly transmissible viruses and the most common cause of diarrhea and vomiting. While illness in most infected persons is relatively mild and self-limiting, severe complications and death may ensue in risk groups, including the extremes of age and persons with reduced immunocompetence due to a range of diseases and treatments. NoVs are diverse, and have evolved into genogroups, genotypes and lineages, with genogroup II (GII) viruses and particularly genogroup II genotype 4 (GII4) viruses as the most prevalent causes of reported diarrheal disease outbreaks in humans. The global dominance of genotype GII4 virus disease may be related to the enhanced transmissibility of these viruses compared with other $\mathrm{NoV}$ genotypes in healthcare settings, resulting in explosive outbreaks, widespread infection and immune selection of escape variants. NoVs, like other RNA viruses, have a replication machinery that is not failure proof, leading to accumulation of mutations in progeny viruses within infected individuals. An additional source of genetic flexibility of NoVs is recombination between related viruses, typically within a genogroup. Recombination can occur when a person is infected simultaneously with two different NoVs, as is common in foodborne outbreaks in which food was contaminated during production (oysters, berries, among others). Such foodborne disease outbreaks may seed viruses across the globe, as the food market is rapidly globalizing, and the trend toward consumption of unprocessed foods adds to the increased risk of outbreaks associated with fresh produce. The population level impact of NoVs is therefore shaped by this

In view of the high burden of disease, the disrupting outbreaks, impact of $\mathrm{NoVs}$ in risk groups, vaccines and antiviral drugs are being developed. For this, understanding the relationship between viral diversity, infection and transmission is crucial. Immunity to NoVs infection appears to be short-lived and the presence of antibodies to the receptor-binding domain of $\mathrm{NoVs}$ is correlated with protection, suggesting that vaccines would need to incorporate antigens from the most common and recent NoVs to be effective. Given the dynamic nature of $\mathrm{NoVs}$ evolution, this is a challenge.

The field has been hampered by the lack of reliable cell culture models, but recent breakthroughs [1] have paved the way for a new and exciting series of research studies. This also extreme genetic flexibility.

\section{KEYWORDS}

- next-generation sequencing $\bullet \mathrm{NoV}$ tracking

"We expect to see the careful application of this technology help solve many of the mysteries of norovirus." 


\section{"In-depth profiling of the viral diversity within single patients on specific treatment regimens can help to identify relevant sites associated, for instance, with immune escape or treatment resistance."}

applies for next-generation sequencing (NGS), allowing profiling of the viral diversity in a range of sample types and in comparison with the rest of the virome and microbiome that may influence the transmission of and expression of disease caused by NoVs. Here, we discuss the potential uses of NGS to address outstanding clinical, public health and research questions in the NoVs field.

\section{NGS methods for NoVs sequencing}

There are currently two popular methods of $\mathrm{NoV}$ NGS: specifically primed amplicon sequencing and agnostic, randomly primed sequencing. Agnostic methods require no prior knowledge of the virus sequence, hence the term. Amplicon sequencing uses forward and reverse primers placed across the virus genome for reverse transcription and PCR amplification. The amplicons overlap and, when pooled and sequenced, provide short read coverage across the virus genome. An advantage is that the method focuses sequencing resources on the target virus (which should be mostly NoV if the primers are designed well). The disadvantage is that the method reveals only target viruses, while co-infecting viruses not recognized by the primers are invisible. Examples of amplicon method include a five (or six) amplicon method used by the J Craig Venter Institute (details can be found in GenBank entries, e.g., KC409317); a 22 amplicon method [2] and a three amplicon method [3] as well as amplicon methods for subgenomic NGS [4]. As an alternative, agnostic sequencing uses random primers, typically hexamers, for priming reverse transcription to convert viral RNA into cDNA then PCR to amplify all nucleic acids in a sample. Agnostic methods include protocols to describe the fecal virome [5], random primed RNAseq methods [6] and recently a random primed amplification followed by NoV-specific capture array [7]. Many variations exist [8-13]. These methods benefit from prepurification to remove bacterial, food and host nucleic acid. A big advantage is that all viruses (above a sensitivity threshold) can be represented in the resulting sequences, providing important co-infection data for clinical samples. Also, unexpected $\mathrm{NoV}$ variants that specific primers would not recognize can be amplified and sequenced [14]. The disadvantage is that the short read data can be dominated by non-NoV sequences and assembly/analysis requires special attention. However, efficient algorithms are available to simplify these problems. Sensitivity can be comparable with amplicon methods depending on the level of multiplexing and sample coverage.

\section{Clinical \& public health applications}

An important application of NGS for clinical and public health work is the ability to track outbreaks and identify sources of introduction. Pathogen genomic analysis using Sanger sequencing has been used quite extensively in the NoVs field, and has provided deep insights into the molecular epidemiology. A minimum capsid gene region of $\mathrm{NoV}$ (nucleotides 900-1400) is sufficient to identify a novel virus and place it within known $\mathrm{NoV}$ evolutionary space [15] and NGS is not needed for this kind of task. Also, identifying patients with a near identical sequence of the minimum capsid gene region has been used to link cases to an outbreak and for practical reasons has been workable. However, given the evolutionary rate of $\mathrm{NoV}$, the resolution for tracking a virus through a care facility or hospital to the level of individual transmission events (who infected whom) is of course greater with full genomes compared with the genotyping fragment.

An important consideration when using NGS for tracking the origin of a NoV infection is the choice of a reference sequence database. Ideally, routine staff sampling/sequencing as well as a subset sampling/sequencing of the local diagnostic clinic (e.g., every 50th diarrhea sample processed in the clinic over the past 3 years) would provide such a reference database. In studies of nosocomial outbreaks, sequencing of samples from patients at the moment of hospitalization can be used as a proxy for community sampling [16]. If such sampling/sequencing were performed throughout the country, a detailed picture of $\mathrm{NoV}$ movement would be available to inform public health measures. As an example of the utility of this approach, during the recent Ebola outbreak in west Africa, the availability of a public database of all available genomes made it possible to identify the village from which new cases arose [17-19].

Other potential applications are in environmental virology. Large foodborne outbreaks of NoVs have been linked to contaminated berries, shellfish and produce, all linked to contact with sewage-contaminated water. Molecular testing for NoVs is now accepted as reference method in food virology, and Sanger sequencing has been used to link products to human disease outbreaks. Here, NGS could also provide greater resolution, 
although the biggest challenge will be the level of detection needed to obtain reliable sequence.

\section{Research applications}

With the development of antivirals and vaccines, it is crucial to understand potential escape mechanisms, such as epitope changes and mutations conferring resistance to antiviral drugs. Indepth profiling of the viral diversity within single patients on specific treatment regimens can help to identify relevant sites associated, for instance, with immune escape or treatment resistance.

An interesting future potential lies in the combined data that are increasingly becoming available though metagenomic and virome studies. With the increasing popularity of gut microbiome research, the unbiased sequencing of fecal samples coupled with strategic population-level sampling reveals the diversity of viruses circulating in the sampled population. The same applies for environmental samples, and interesting experiments are ongoing using sewage as surveillance tool. Combining sequence data from systematic stool and environmental sample collections with advanced phylodynamic analysis can inform on expansions of the virus diversity as a proxy for increased circulation in the absence of disease based reporting. Such an approach can also be used to mine metagenomic datasets for novel NoVs. The emergence of recombinant NoVs with nonstructural genes that have not previously been observed in humans raises the question of sources of these genome segments, including the potential of nonhuman reservoirs.

\section{Challenges for implementing NGS in a clinical setting}

NGS can support diagnostics and can provide unexpected insight into the complexity of pathogens in our environment. However, NGS is unlikely to replace current rigorous diagnostic algorithms with known positive and negative predictive values in the near future unless some important hurdles are overcome. One limitation is speed. Currently most sequencing facilities, library preparation, sequencing instruments and possibly downstream analysis teams are shared by a number of applications including tumor genotyping, bacterial typing, microbiome work, among others. If NGS is to be a useful tool to influence clinical care, a rapid 24-48-h turnaround from sample to full genome phylogenetic analysis is needed. Such turnaround was possible during the Ebola epidemic [18] but did require dedicated staff and facilities. Sample preps, amplification, library preps can be performed in less than $18 \mathrm{~h}$, actual sequencing runtime can be $6-12 \mathrm{~h}$ or less depending on the instrument. Data assembly into genomes and phylogenetic analyses can be performed in a few hours depending on the level of analysis and the number of new genomes, among others. If existing genomes are available from the hospital, staff and community, adding 20-30 new genomes from an outbreak could be performed within a working day to provide an indication of the origin of the current infections.

A second limitation may be cost. Currently, a sample processed for NGS will cost US \$100-200 for the sample amplification, library prep and sequencing costs. Downstream analysis costs will include bioinformatics support, and computational and data storage resources. Whether these costs are justified will depend on the clinical need and utility of the data provided. For surveillance, the idea of applying agnostic NGS routinely to a subset of fecal samples coming through a diagnostic clinic might be an effective tool for monitoring diagnostic performance, and diversity of known pathogens and for detection of potential pathogens not covered by targeted diagnostics.

The sensitivity of NGS means that a few genomes in the sample will contribute to the sequence data in the final set. This is great for sequencing of low abundance viruses but it also means that cross contamination between samples and between projects can occur and may compromise the use of the data for clinical interpretations. Methods to limit crosstalk are similar to the strict rules applied in molecular diagnostic facilities, maintaining strict barriers between the workflows from molecular biology labs involved in cell culture growth of viruses or cloning and clinical laboratories. To reduce false positives from contamination, an archive of all sequencing projects should be available for follow-up of unexpected sequence content. Repeat sequencing on freshly prepared samples, confirmation with alternate methods, phylogenetic analysis that show the expected sequence evolution patterns can all help validate NGS results.

\section{Challenges of communicating phylogenetics to public health officials}

Useful guidance comes from the application of genomic epidemiology in legal cases [20], but it is easier to disprove a connection (e.g., a source
"With the increasing popularity of gut microbiome research, the unbiased sequencing of fecal samples coupled with strategic population-level sampling reveals the diversity of viruses circulating in the sampled population.” 
of virus for a single infection) than to prove such a link conclusively. Even with known direct transmission between two patients, the viruses continue to evolve in each patient and viruses can differ when sequenced. Also, each patient may harbor a quasispecies of viruses and two samplings from the same patient may yield two slightly different sequences, complicating interpretation. Third, NGS protocols and data analyses have not yet been standardized to the degree of rigor needed in accredited clinical or food microbiology laboratories. Nonetheless, NGS data have been used to determine the origin of the virus from outbreaks and to evaluate control measures. This can only be done by careful design of study questions, with crucial cooperation between clinicians, laboratory experts and public health experts. For the time being, this requires a research mindset, in order to ensure the careful validation of these novel methods.
Given the ease of generating full genomes from small amounts of clinical diagnostic samples and falling prices of NGS methods, generating sequence data from well-designed large sample studies is now feasible. We expect to see the careful application of this technology help solve many of the mysteries of NoV.

\section{Financial \& competing interests disclosure}

This work was supported by funding from $Z O N M W$, The Netherlands under grant agreement 91213058 , and by the Horizon 2020 program of the European Commission under grant agreement 643476 (COMPARE; www.compareeurope.eu). The authors have no other relevant affiliations or financial involvement with any organization or entity with a financial interest in or financial conflict with the subject matter or materials discussed in the manuscript apart from those disclosed.

No writing assistance was utilized in the production of this manuscript.

\section{References}

1 Ettayebi K, Crawford SE, Murakami K et al. Replication of human noroviruses in stem cell-derived human enteroids. Science 353(6306), 1387-1393 (2016).

2 Kundu S, Lockwood J, Depledge DP et al. Next-generation whole genome sequencing identifies the direction of noroviruses transmission in linked patients. Clin. Infect. Dis. 57(3), 407-414 (2013).

3 Cotten M, Petrova V, Phan MV et al. Deep sequencing of noroviruses genomes defines evolutionary patterns in an urban tropical setting. J. Virol. 88(19), 11056-11069 (2014).

4 Bull RA, Eden JS, Luciani F, Mcelroy K, Rawlinson WD, White PA. Contribution of intra- and interhost dynamics to norovirus evolution. J. Virol. 86(6), 3219-3229 (2012).

5 Cotten M, Oude Munnink B, Canuti M et al. Full genome virus detection in fecal samples using sensitive nucleic acid preparation, deep sequencing, and a Novel iterative sequence classification algorithm. PLoS ONE 9(4), e93269 (2014).

6 Batty EM, Wong TH, Trebes A et al. A modified RNA-Seq approach for whole genome sequencing of RNA viruses from faecal and blood samples. PLoS ONE 8(6), e66129 (2013).

7 Brown JR, Roy S, Ruis C et al. Norovirus whole genome sequencing by SureSelect target enrichment: a robust and sensitive method. J. Clin. Microbiol. 54(10), 2530-2537 (2016).

8 Wong TH, Dearlove BL, Hedge J et al. Whole genome sequencing and de novo assembly identifies Sydney-like variant noroviruses and recombinants during the winter 2012/2013 outbreak in England. Virol. J. 10, 335 (2013).

9 de Vries M, Oude Munnink BB, Deijs M et al. Performance of VIDISCA-454 in feces-suspensions and serum. Viruses 4(8), 1328-1334 (2012).

10 Moore NE, Wang J, Hewitt J et al. Metagenomic analysis of viruses in feces from unsolved outbreaks of gastroenteritis in humans. J. Clin. Microbiol. 53(1), 15-21 (2015).

11 Hasing ME, Hazes B, Lee BE, Preiksaitis JK, Pang XL. A next generation sequencing-based method to study the intra-host genetic diversity of $\mathrm{NoV}$ in patients with acute and chronic infection. BMC Genomics 17, 480 (2016).

12 Smits SL, Schapendonk CM, van Beek J et al. New viruses in idiopathic human diarrhea cases, The Netherlands. Emerg. Infect. Dis. 20(7), 1218-1222 (2014).

13 Svraka S, Rosario K, Duizer E, van der Avoort H, Breitbart M, Koopmans M. Metagenomic sequencing for virus identification in a public-health setting. J. Gen. Virol. 91(11), 2846-2856 (2010).
14 De Graaf M. Norovirus infection in harbour porpoises. Emerg. Infect. Dis. (2016) (In Press).

15 Verhoef L, Williams KP, Kroneman A et al. Selection of a phylogenetically informative region of the norovirus genome for outbreak linkage. Virus Genes 44(1), 8-18 (2012).

16 Sukhrie FH, Beersma MF, Wong A et al. Using molecular epidemiology to trace transmission of nosocomial $\mathrm{NoV}$ infection. J. Clin. Microbiol. 49(2), 602-606 (2011).

17 Smits SL, Pas SD, Reusken CB et al. Genotypic anomaly in Ebola virus strains circulating in Magazine Wharf area, Freetown, Sierra Leone, 2015. Euro. Surveill. 20(40)doi:10.2807/1560-7917. ES.2015.20.40.30035 (2015). www.eurosurveillance.org/

18 Arias A, Watson SJ, Asogun D et al. Rapid outbreak sequencing of Ebola virus in Sierra Leone identifies transmission chains linked to sporadic cases. Virus Evol. 2(1), (2016). http://dx.doi.org/10.1093/ve/vew016

19 Diallo B, Sissoko D, Loman NJ et al. Resurgence of Ebola virus disease in Guinea linked to a survivor with virus persistence in seminal fluid for more than 500days. Clin. Infect. Dis 63(10), 1353-1356 (2016).

20 Vandamme AM, Pybus OG. Viral phylogeny in court: the unusual case of the Valencian anesthetist. BMC Biol. 11, 83 (2013). 